\title{
Thyroid transcription factor-1, but not p53, is helpful in distinguishing moderately differentiated neuroendocrine carcinoma of the larynx from medullary carcinoma of the thyroid
}

\author{
Michelle S Hirsch ${ }^{1}$, William C Faquin ${ }^{2}$ and Jeffrey F Krane ${ }^{1}$ \\ ${ }^{1}$ Department of Pathology, Brigham and Women's Hospital and Harvard Medical School, Boston, MA, USA \\ and ${ }^{2}$ Department of Pathology, Massachusetts General Hospital and Harvard Medical School, Boston, MA, \\ $U S A$
}

\begin{abstract}
Moderately differentiated neuroendocrine carcinoma/atypical carcinoid tumor is the most common nonsquamous malignancy in the larynx; however, due to morphologic overlap and calcitonin immunoreactivity, it can be difficult to distinguish from thyroid medullary carcinoma. Currently, low serum calcitonin is the most reliable means for distinguishing primary laryngeal moderately differentiated neuroendocrine carcinoma from metastatic medullary carcinoma. Thyroid transcription factor-1 (TTF-1) is positive in at least $80 \%$ of medullary carcinomas, but has not been evaluated in laryngeal moderately differentiated neuroendocrine carcinomas. Additionally, it has been suggested that $\mathrm{p} 53$ is positive in laryngeal moderately differentiated neuroendocrine carcinomas and negative in other neuroendocrine tumors, but this has not been validated. The purpose of this study was to determine if the immunohistochemical markers TTF-1 and p53 could be used to discriminate between laryngeal moderately differentiated neuroendocrine carcinomas and thyroid medullary carcinomas. Eight laryngeal moderately differentiated neuroendocrine carcinomas and $\mathbf{1 0}$ thyroid medullary carcinomas were identified from the archival files of the BWH and MGH Pathology Departments. Hematoxylin and eosin slides were reviewed, and immunohistochemistry was performed using antibodies to calcitonin, TTF-1, and p53. Calcitonin immunohistochemistry demonstrated immunoreactivity in $100 \%$ of laryngeal moderately differentiated neuroendocrine carcinomas $(N=8)$ and $100 \%$ of thyroid medullary carcinomas $(N=10)$. There was weak, focal immunoreactivity with TTF-1 in one of eight (13\%) laryngeal moderately differentiated neuroendocrine carcinomas, whereas nine of ten $(90 \%)$ medullary carcinomas were positive for TTF-1, with strong diffuse staining in seven of these cases $(78 \%)$. p53 was positive in three of six $(50 \%)$ laryngeal moderately differentiated neuroendocrine carcinomas, and three of ten $(30 \%)$ medullary carcinomas. Our data demonstrate that immunoreactivity for TTF-1, but not calcitonin or p53, may be helpful in distinguishing laryngeal moderately differentiated neuroendocrine carcinoma and thyroid medullary carcinoma. In particular, diffuse and/or strong TTF-1 immunoreactivity favors a diagnosis of primary thyroid medullary carcinoma over laryngeal moderately differentiated neuroendocrine carcinoma.
\end{abstract}

Modern Pathology (2004) 17, 631-636, advance online publication, 16 April 2004; doi:10.1038/modpathol.3800105

Keywords: carcinoid; neuroendocrine; medullary; larynx; thyroid; TTF-1; p53; calcitonin

Correspondence: JF Krane, MD, PhD, Department of Pathology, Brigham and Women's Hospital, 75 Francis Street, Boston, MA 02115, USA.

E-mail: jfkrane@bics.bwh.harvard.edu

Presented in part at the 92nd annual United States and Canadian Academy of Pathology meeting, March 22-28, 2003, Washington, DC.

Received 21 October 2003; revised 09 February 2004; accepted 16 February 2004; published online 16 April 2004
Moderately differentiated neuroendocrine carcinoma (atypical carcinoid tumor) is the most common nonsquamous malignancy in the larynx, metastasizing to regional lymph nodes, skin, subcutaneous tissue or distant sites in approximately $20-40 \%$ of cases. $^{1,2}$ Moderately differentiated neuroendocrine carcinomas of the larynx and medullary carcinomas of the thyroid demonstrate morphological overlap, and can be microscopically indistinguishable, particularly when presenting as a metastasis. Currently, 
serum calcitonin is the only objective means for distinguishing primary moderately differentiated neuroendocrine carcinoma of the larynx from metastatic medullary carcinoma, as calcitonin immunohistochemistry is reportedly positive in both tumors. ${ }^{3-6}$

Thyroid transcription factor-1 (TTF-1) is a nuclear homeodomain transcription factor that is selectively expressed in developing respiratory epithelium, thyroid and diencephelon. ${ }^{7,8}$ Immunohistochemical studies have shown that TTF-1 is expressed in a subset of lung and most thyroid neoplasms, including pulmonary neuroendocrine tumors and medullary carcinoma of the thyroid. ${ }^{9-12}$ Among pulmonary neuroendocrine tumors, positive expression of TTF-1 varies from 35 to $95 \%$ in well-differentiated neuroendocrine tumors ('typical' carcinoids), , $^{9,12}$ and $75-100 \%$ in atypical carcinoids, large-cell neuroendocrine carcinomas and small-cell carcinomas. $^{9}$ In thyroid medullary carcinomas, TTF-1 immunoreactivity has been shown to be consistently positive. ${ }^{10-13}$

Despite the overlapping morphologic and immunophenotypic features of moderately differentiated neuroendocrine carcinomas of the larynx and medullary carcinomas of the thyroid, TTF-1 expression in laryngeal moderately differentiated neuroendocrine carcinomas has not been examined. The objective of this study therefore was to determine if immunohistochemical staining for TTF-1 could be used to discriminate between moderately differentiated neuroendocrine carcinomas of the larynx and thyroid medullary carcinomas. p53 was also studied as a potential discriminator as at least one prior investigation has suggested that it may be a useful marker for moderately differentiated neuroendocrine carcinomas. ${ }^{5}$

\section{Materials and methods}

Cases were obtained from the archival files in the Departments of Pathology at Brigham and Women's Hospital and Massachusetts General Hospital, Boston, MA, USA. Eight moderately differentiated neuroendocrine carcinomas of the larynx (five male and three female patients; mean age 61 years, range 45-82 years) and 10 thyroid medullary carcinomas (six male and four female patients; mean age 49 years, range 28-79 years) were evaluated. Hematoxylin and eosin-stained slides generated from formalin-fixed, paraffin-embedded tissue were reviewed to confirm the diagnoses prior to inclusion in the study. Immunohistochemistry was performed using the Envision Plus/Horseradish Peroxidase system (DAKO Corp., Carpinteria, CA, USA), a rabbit antibody to calcitonin (1:8000; DAKO), a mouse monoclonal antibody to TTF-1 (clone 8G7G3/1; 1:100; DAKO), and a mouse monoclonal antibody to p53 (clone DO-1; 1:100, Immunotech, Miami, FL, USA). Briefly, paraffin-embedded sections were incubated in hydrogen peroxidase and absolute alcohol for $30 \mathrm{~min}$ to block endogenous peroxidase activity. Antigen retrieval was performed prior to incubation with the TTF-1 and p53 antibodies using microwave pretreatment in a citrate buffer $(\mathrm{pH}=6.0)$. Antigen retrieval was not performed prior to incubation with the calcitonin antibody. Tissue sections were subsequently incubated with a primary antibody for $40-60 \mathrm{~min}$ at $25^{\circ} \mathrm{C}$. Following TBS rinses, the tissue was incubated using the Envision Plus secondary antibody (either anti-rabbit or anti-mouse) for $30 \mathrm{~min}$ followed by diaminobenzidine for $5 \mathrm{~min}$ (calcitonin) or $10 \mathrm{~min}$ (TTF-1 and p53). Appropriate positive and negative controls (using normal rabbit or mouse serum) were stained in parallel. Calcitonin and TTF-1 were evaluated for positive cytoplasmic and nuclear staining, respectively. p53 was considered positive if $>1 \%$ of tumor cells demonstrated a nuclear immunostaining pattern.

\section{Results}

Histologically, moderately differentiated neuroendocrine carcinomas of the larynx $(N=8)$ and medullary carcinomas of the thyroid $(N=10)$ had overlapping features (Figure $1 \mathrm{a}$ and b), which included: epithelioid to spindle cells with moderate amounts of pale eosinophilic cytoplasm; an architectural arrangement in cords, nests and solid sheets; characteristic nuclei with stippled neuroendocrine-type chromatin; scattered mitoses; and a prominent vascular network. Two of the laryngeal tumors and five of the thyroid tumors were metastatic to regional lymph nodes. Immunohistochemistry with calcitonin, TTF-1 and p53 was performed on all primary tumors (Table 1), one metastatic laryngeal moderately differentiated neuroendocrine carcinoma and three metastatic medullary carcinomas of the thyroid.

\section{Calcitonin Immunostaining}

Immunohistochemistry with calcitonin demonstrated diffuse cytoplasmic immunoreactivity in eight of eight $(100 \%)$ primary moderately differentiated neuroendocrine carcinomas of the larynx and 10 of $10(100 \%)$ primary medullary carcinomas of the thyroid.

\section{TTF-1 Immunostaining}

There was weak, focal nuclear immunoreactivity with TTF-1 in one of eight $(13 \%)$ moderately differentiated neuroendocrine carcinomas of the larynx; the remainder of cases were negative (Figure 1c). In contrast, nine of $10 \quad(90 \%)$ medullary carcinomas were positive for TTF-1 with seven of these cases $(78 \%)$ demonstrating a strong diffuse nuclear staining pattern (Figure 1d). The one TTF-1negative medullary carcinoma was more poorly differentiated with a spindle cell morphology. 

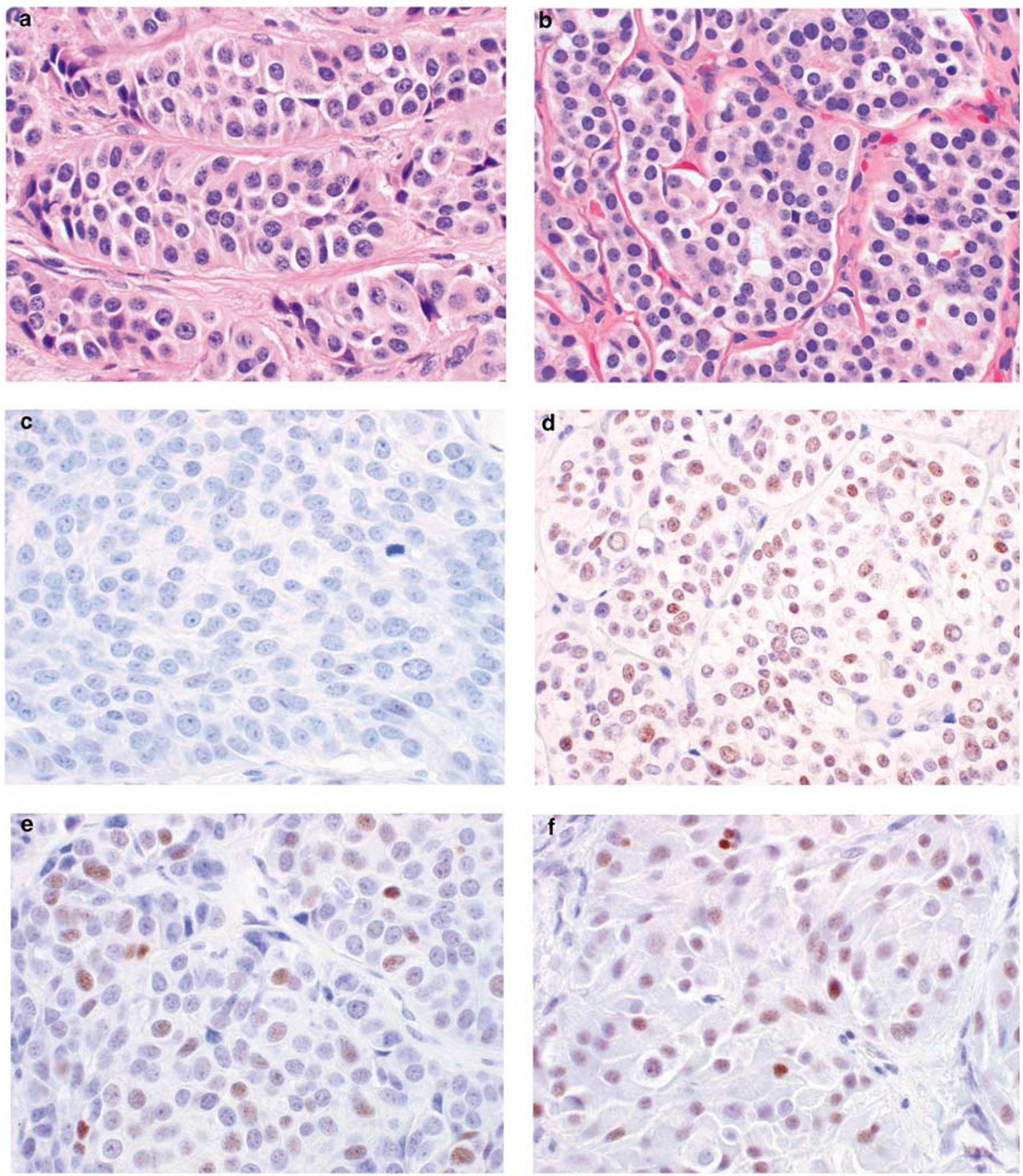

Figure 1 Moderately differentiated neuroendocrine carcinomas of the larynx (a) and medullary carcinomas of the thyroid (b) are indistinguishable by morphology alone (H\&E stain). The majority of moderately differentiated neuroendocrine carcinomas of the larynx are negative for TTF-1 (c), whereas most medullary carcinomas of the thyroid demonstrate positive nuclear staining for TTF-1 (d). Occasional cells demonstrate nuclear positivity for p53 in a subset of moderately differentiated neuroendocrine carcinomas of the larynx (e) and medullary carcinomas of the thyroid (f).

\section{p53 Immunostaining}

p53 was considered positive if $>1 \%$ of tumor cells demonstrated nuclear staining. Six of the eight primary laryngeal moderately differentiated neuroendocrine carcinomas were available for p53 immunohistochemistry: three of these six cases (50\%) were positive for p53 (Figure 1e). Three of
$10(30 \%)$ medullary carcinomas of the thyroid were immunoreactive for p53 (Figure 1f).

\section{Discussion}

Neuroendocrine carcinomas of the larynx are infrequent, yet well-recognized tumors that represent 
Table 1 Immunohistochemistry results in primary moderately differentiated neuroendocrine carcinomas of the larynx and primary medullary carcinomas of the thyroid

\begin{tabular}{|c|c|c|c|}
\hline & \multicolumn{3}{|c|}{ Antibody [\#pos cases/\#total cases (\% positive)] } \\
\hline & Calcitonin & $T T F-1$ & p53 \\
\hline MDNECs of the larynx & $8 / 8(100 \%)$ & $1 / 7(14 \%)$ & $3 / 6(50 \%)$ \\
\hline MCs of the thyroid & $10 / 10(100 \%)$ & $9 / 10(90 \%)$ & $3 / 10(30 \%)$ \\
\hline
\end{tabular}

MDNECs = moderately differentiated neuroendocrine carcinomas; MCs = medullary carcinomas.

the second most common laryngeal malignancy after squamous cell carcinoma. Goldman et $a 1^{14}$ described the first atypical carcinoid in 1969. Subsequently, small cell (oat cell) carcinoma and typical carcinoid tumor of the larynx were described by Olofsson and van Nostrand ${ }^{15}$ in 1972, and Markel et $a l^{16}$ in 1980, respectively. Although the morphologic findings of these tumors have not varied, the nomenclature of laryngeal neuroendocrine neoplasms has changed since the time that they were first recognized. ${ }^{2-4,6,17,18}$

In 1993, the World Health Organization (WHO) subdivided laryngeal neuroendocrine tumors into carcinoid, atypical carcinoid and small cell carcinoma. ${ }^{17}$ More recently, it has been suggested ${ }^{2}$ that the following nomenclature would more accurately represent morphologic and biologic differences between laryngeal neuroendocrine neoplasms: well-differentiated neuroendocrine carcinoma (typical carcinoid), moderately differentiated neuroendocrine carcinoma (atypical carcinoid), poorly differentiated neuroendocrine carcinoma-small cell type and poorly differentiated neuroendocrine carcinoma-large cell type. Most pertinent to this study, the change in nomenclature from atypical carcinoid to moderately differentiated neuroendocrine carcinoma would accurately reflect the fact that these tumors are aggressive, malignant neoplasms, which have a significant potential to metastasize.

Morphologically, moderately differentiated neuroendocrine carcinomas of the larynx are most commonly composed of nests or sheets of epithelioid cells with characteristic neuroendocrine features including round to oval nuclei, stippled chromatin, occasional nucleoli and variable hyperchromasia. Pleomorphism, mitoses, necrosis and an infiltrative border are more often appreciated when compared to well-differentiated neuroendocrine carcinomas (carcinoid tumors). The most troubling differential diagnosis for moderately differentiated neuroendocrine carcinoma of the larynx (especially those that are metastatic) is medullary carcinoma of the thyroid, as morphologically these two tumors can be indistinguishable. Additionally, immunohistochemistry has not proven to be useful in making this distinction since both tumors are immunoreac- tive with keratins, neuroendocrine markers, CEA and calcitonin..$^{1,4,6}$

Currently, the only known objective discriminator between moderately differentiated neuroendocrine carcinoma of the larynx and medullary carcinoma of the thyroid is serum calcitonin, which should be elevated in medullary carcinomas of the thyroid and within normal limits in moderately differentiated neuroendocrine carcinomas of the larynx. However, there are rare exceptions as three out of over 300 reported cases of moderately differentiated neuroendocrine carcinoma of the larynx in the literature were reportedly associated with an elevated level of calcitonin. ${ }^{19-21}$ In each of these three cases, thyroidectomies were performed and the evaluated tissue was reportedly negative for medullary carcinoma. Despite the recognition that only very rare cases of moderately differentiated neuroendocrine carcinoma of the larynx may have elevated serum calcitonin levels, a marker at the light microscopic level would be helpful for objectively distinguishing moderately differentiated neuroendocrine carcinoma of the larynx from medullary carcinoma of the thyroid.

Mutations of the p53 gene have been commonly associated with numerous malignancies. More specifically, a point mutation causes a conformational change in the p53 protein, which increases the halflife and results in an accumulation of protein within neoplastic cells, which can then be detected by immunohistochemical analysis. Studies have shown that overexpression of p53 in neuroendocrine tumors, including medullary carcinomas of the thyroid, is rare. ${ }^{22,23}$ In contrast, it has been suggested that p53, which has been shown to be diffusely positive in three laryngeal moderately differentiated neuroendocrine carcinomas, may play a role in the pathogenesis of this tumor, unlike other neuroendocrine tumors. ${ }^{5}$ However, these findings have not been validated.

TTF-1 is a transcription factor that regulates the gene expression of thyroid-specific proteins, including thyroglobulin, thyrotropin receptor and thyroperoxidase. TTF-1 has also been shown to regulate surfactant expression in lung as it is expressed in type II pneumocytes and Clara cells. Multiple studies have shown that TTF-1 is immunoreactive in both neuroendocrine and non-neuroendocrine 
tumors of the thyroid and lung. In the thyroid, $80-100 \%$ of follicular adenomas, follicular carcinomas, papillary carcinomas, and medullary carcinomas are positive for TTF-1. ${ }^{10-13}$ Adenocarcinomas of the lung are positive in approximately $70-80 \%$ of cases, and squamous cell carcinomas of the lung are nearly always negative. ${ }^{24-26}$ In contrast, neuroendocrine tumors of the lung demonstrate variable amounts of TTF-1 immunoreactivity with the least amount of staining in carcinoid tumors $(\sim 35 \%$ of cases), and the greatest amount in atypical carcinoid tumors and small cell carcinomas (greater than $90 \%$ of cases) ${ }^{9,26}$ However, TTF-1 has not been evaluated in laryngeal neuroendocrine carcinomas.

In the present study, TTF-1, calcitonin and p53 expression are compared between moderately differentiated neuroendocrine carcinomas of the larynx and medullary carcinomas of the thyroid. Our findings confirm that immunohistochemistry with calcitonin does not discriminate between moderately differentiated neuroendocrine carcinoma of the larynx and medullary carcinoma of the thyroid as it was identified in $100 \%$ of each of these tumor types. Additionally, p53 does not appear to be a useful marker for making this distinction, since it was found in $50 \%$ of moderately differentiated neuroendocrine carcinomas of the larynx and 30\% of medullary carcinomas of the thyroid.

In contrast, TTF-1 immunostaining does appear to be helpful in distinguishing these two tumors, as it is present in only $13 \%$ of moderately differentiated neuroendocrine carcinomas of the larynx (one of eight cases), and in $90 \%$ of medullary carcinomas of the thyroid (nine of 10 cases). It should be noted, however, that the one TTF-1-positive moderately differentiated neuroendocrine carcinoma case demonstrated weak, focal positivity, whereas seven of nine medullary carcinomas were strongly and diffusely positive for this marker. Additionally, the only medullary carcinoma that was negative for TTF-1 was a poorly differentiated variant with a spindle cell morphology.

Two of the laryngeal tumor cases and five of the thyroid tumor cases were also metastatic to regional lymph nodes. One of the two metastatic moderately differentiated neuroendocrine carcinomas of the larynx was available for immunostaining. In this case, both the primary and metastatic tumors were positive for calcitonin and negative for TTF-1. p53 was only performed on the metastatic tumor and was positive. In contrast, three of the five metastatic medullary carcinomas were stained: in two of these cases, calcitonin, TTF-1 and p53 were positive in both the primary and metastatic sites; however, in the third case, the primary tumor was positive for calcitonin and TTF-1 and negative for p53, whereas the metastatic tumor was only positive for calcitonin and negative for both TTF-1 and p53. We recognize that the number of metastatic tumors evaluated in this series is limited. Therefore, to further support the use of TTF-1 staining in metastatic neuroendo- crine tumors of unknown primary, especially to lymph nodes and cutaneous sites in the head and neck region, additional cases will need to be examined. Still, these limited results appear to support the use of TTF-1 in distinguishing between moderately differentiated neuroendocrine carcinoma of the larynx and medullary carcinoma of the thyroid.

Overall, the findings suggest that diffuse and/or strong TTF-1 immunoreactivity, in combination with positive calcitonin staining, favors a diagnosis of medullary carcinoma of the thyroid over moderately differentiated neuroendocrine carcinoma of the larynx. While not completely discriminatory, the combined use of calcitonin and TTF-1 in the evaluation of a metastatic neuroendocrine carcinoma of unknown primary in the head and neck region can be a useful first step in guiding the clinical evaluation of these tumors.

\section{Acknowledgements}

We thank Dr Geraldine Pinkus and Ms Catherine Quigley for their technical assistance with immunohistochemical studies.

\section{References}

1 Woodruff JM, Senie RT. Atypical carcinoid tumor of the larynx: a critical review of the literature. ORL J Otorhinolaryngol Relat Spec 1991;53:194-209.

2 Mills SE. Neuroectodermal neoplasms of the head and neck with emphasis on neuroendocrine carcinomas. Mod Pathol 2002;15:264-278.

3 Woodruff JM, Huvos AG, Erlandson RA, et al. Neuroendocrine carcinomas of the larynx. Am J Surg Pathol 1985;9:771-790.

4 Wenig BM, Hyams VJ, Heffner DK. Moderately differentiated neuroendocrine carcinoma of the larynx. Cancer 1988;62:2658-2676.

5 McCluggage WG. Atypical carcinoid tumor of the larynx: an immunohistochemical, ultrastructural, and flow cytometric analysis. Ultrastruct Pathol 1997;21: 431-438.

6 Ferlito A, Barnes L, Rinaldo A, et al. A review of neuroendocrine neoplasms of the larynx: update on diagnosis and treatment. J Laryngol Otol 1998; 112:827-834.

7 Guazzi S, Price M, de Felice M, et al. Thyroid nuclear factor 1 (TTF-1) contains a homeodomain and displays a novel DNA binding specificity. EMBO J 1990;9: 3631-3639.

8 Lazzaro D, Price M, de Felice M, et al. The transcription factor TTF-1 is expressed at the onset of thyroid and lung morphogenesis and in restricted regions of the foetal brain. Development 1991;113:1093-1104.

9 Folpe AL, Gown Am, Lamps LW, et al. Thyroid transcription factor-1: immunohistochemical evaluation in pulmonary neuroendocrine tumors. Mod Pathol 1999;12:5-8.

10 Katoh R, Miyagi E, Nakamura N, et al. Expression of thyroid transcription factor-1 (TTF-1) in human $\mathrm{C}$ cells 
and medullary thyroid carcinomas. Hum Pathol 2000;31:386-393.

11 Kaufmann O, Dietel M. Expression of thyroid transcription factor-1 in pulmonary and extrapulmonary small cell carcinomas and other neuroendocrine carcinomas of various primary sites. Histopathology 2000;36:415-420.

12 Oliveira AM, Tazelaar HD, Myers JL, et al. Thyroid transcription factor-1 distinguishes metastatic pulmonary from well-differentiated neuroendocrine tumors of other sites. Am J Surg Pathol 2001;25: 815-819.

13 Agoff SN, Lamps LW, Philip AT, et al. Thyroid transcription factor-1 is expressed in extrapulmonary small cell carcinomas but not in other extrapulmonary neuroendocrine tumors. Mod Pathol 2000;13:238-242.

14 Goldman NC, Hood CI, Singleton GT. Carcinoid of the larynx. Arch Otolaryngol 1969;90:91-93.

15 Olofsson J, van Nostrand AWP. Anaplastic small cell carcinoma of the larynx. Case report. Ann Otol Rhinol Laryngol 1972;81:284-287.

16 Markel SF, Magielski JE, Beals TF. Carcinoid tumor of the larynx. Arch Otolaryngol 1980;106:777-778.

17 Ferlito A. The World Health Organization's revised classification of tumors of the larynx, hypopharynx, and trachea. Ann Otol Rhinol Laryngol 1993;102: 666-669.

18 Mills SE. Neuroendocrine tumors of the head and neck: a selected review with emphasis on terminology. Endocrinol Pathol 1996;7:329-343.
19 Sweeney EC, McDonnell L, O’Brien C. Medullary carcinoma of the thyroid presenting as tumors of the pharynx and larynx. Histopathology 1981;5:263-275.

20 Smets G, Warson F, Dehou M-F, et al. Metastasizing neuroendocrine carcinoma of the larynx with calcitonin and somatostatin secretion and CEA production, resembling medullary thyroid carcinoma. Virchows Arch 1990;416:539-543.

21 Insabato L, De Rosa G, Terracciano LM, et al. A calcitonin-producing neuroendocrine tumor of the larynx: a case report. Tumori 1993;79:227-230.

22 Holm R, Nesland JM. Retinoblastoma and p53 tumor suppressor gene protein expression in carcinomas of the thyroid gland. J Pathol 1994;172:267-272.

23 Wang DG, Johnston CF, Anderson N, et al. Overexpression of the tumor suppressor gene p53 is not implicated in neuroendocrine carcinogenesis. J Pathol 1995;175:397-401.

24 Pelosi G, Fraggetta F, Pasini F, et al. Immunoreactivity for thyroid transcription factor-1 in stage I non-small cell carcinomas of the lung. Am J Surg Pathol 2001; 25:363-372.

25 Yatabe Y, Mitsudomi T, Takahashi T. TTF-1 expression in pulmonary adenocarcinomas. Am J Surg Pathol 2002;26:767-773.

26 Wu M, Wang B, Gil J, et al. p63 and TTF-1 immunostaining. A useful marker panel for distinguishing small cell carcinoma of lung from poorly differentiated squamous cell carcinoma of lung. Am J Clin Pathol 2003;119:696-702. 